\title{
Novel Trajectory Optimization Algorithm of Vehicle-borne LiDAR Mobile Measurement System
}

\author{
Ming Guo, ${ }^{1,2,3,4}$ Mengxi Sun, ${ }^{1}$ Tengfei Zhou, ${ }^{1}$ \\ Bingnan Yan, ${ }^{1}$ Yuquan Zhou, ${ }^{1}$ and Deng Pan $^{5 *}$ \\ ${ }^{1}$ School of Geomatics and Urban Spatial Informatics, Beijing University of Civil Engineering and Architecture, \\ Beijing 102616, China \\ ${ }^{2}$ Engineering Research Center of Representative Building and Architectural Heritage Database, \\ Ministry of Education, Beijing 100044, China \\ ${ }^{3}$ Key Laboratory of Modern Urban Surveying and Mapping, National Administration of Surveying, \\ Mapping and Geoinformation, Beijing 100044, China \\ ${ }^{4}$ Beijing Key Laboratory for Architectural Heritage Fine Reconstruction \& Health Monitoring, \\ Beijing 102616, China \\ ${ }^{5}$ School of Humanity and Law, Beijing University of Civil Engineering and Architecture, Beijing 102616, China
}

(Received August 5, 2020; accepted October 20, 2020)

Keywords: vehicle-borne LiDAR measurement system, smooth trajectory, point cloud accuracy improvement, B-spline curve, adaptive segmentation

Since a vehicle-borne light detection and ranging (LiDAR) measurement system is affected by the signal shielding and rolling vibration of the vehicle as it moves, commonly available trajectory data are usually low-quality data with noise. Although the position and attitude data of the trajectory are processed by joint Kalman filtering, there are still fluctuations in local areas, which require processing to smooth the acquired trajectory data. In this paper, a model of vehicle motion is proposed to analyze the trend of a vehicle trajectory over time by recording the vehicle position, velocity, and attitude information in real time. Next, the vehicle motion trajectory is processed in sections to understand the motion state intuitively. Experimental results show that the descriptions of ground features by the vehicle-mounted and ground point clouds are almost the same. The relative accuracy can reach $0.013 \mathrm{~m}$ by selecting multiple spacings for comparison. In conclusion, the segmentation optimization method for vehicle trajectories proposed in this study is expected to provide a higher accuracy than the current techniques used for optimizing the accuracy of vehicle trajectories.

\section{Introduction}

In recent years, with the continuous acceleration of global digitalization, the demand for 3D geospatial information has increased markedly. Vehicle-mounted mobile measurement systems have been employed for studies on the construction of smart cities, ${ }^{(1,2)}$ city transportation programming, ${ }^{(3)}$ the modeling of highways, ${ }^{(4)}$ cadastral surveys, ${ }^{(5)}$ and other fields owing to their high degree of automation, short data generation cycle, low cost, and high flexibility. ${ }^{(6,7)}$ The ever-changing operating environment of vehicle-mounted mobile measurement systems will

*Corresponding author: e-mail: pandeng@bucea.edu.cn

https://doi.org/10.18494/SAM.2020.3052 
have a great impact on data accuracy and may even produce unpredictable errors. Therefore, the point cloud should be modified in addition to performing standard operations to ensure that the mathematical accuracy meets the requirements. In general, it is very important to study how to improve the accuracy of point cloud data of vehicle-mounted mobile measurement systems. ${ }^{(8-10)}$

The vehicle trajectory is an important data obtained by the vehicle light detection and ranging (LiDAR) measurement system, which is jointly determined by the inertial measurement unit (IMU) and global navigation satellite system (GNSS). It includes various motion information such as position, speed, angle, and acceleration. ${ }^{(11)}$ The quality of trajectory data is a key factor affecting the quality of point cloud data. The measurement accuracy of a vehicle mobile measurement system is easily affected by the environment. A design experiment verified that there is a certain rule describing the effects of the LiDAR incident angle and global positioning system (GPS) out-of-lock time on the measurement accuracy of a vehicle-mounted mobile measurement system. ${ }^{(12)}$ Vehicle LiDAR measurement systems are affected when the signal is obscured by vehicle undulation and vibration during movement, which results in the collected trajectory data having a lot of noise. To solve this problem, Liang proposed the modification of the initial registration conversion relationship, including the amounts of rotation and translation for correction, ${ }^{(13)}$ but the registration accuracy of this method only reached the decimeter level. On the basis of such modification, Chen proposed many algorithmic solutions. ${ }^{(14)}$ Their common technologies include point cloud segmentation, target tracking and identification, real-time positioning, and mapping technologies, and the key to the research was how to improve the robustness and scale of the algorithm. Through the research on the processing of a vehicle's 3D trajectory, we conclude that if the overall trajectory data is processed, the amount of calculation is relatively large. After the vehicle motion trajectory is filtered, there is only deviation in local areas; processing all the data will increase the amounts of calculation and waste resources. Therefore, before the trajectory is optimized, it is first segmented. For the onvehicle trajectory turning section, the quadratic rational Bezier curve is used to realize a spatial arc transition, ${ }^{(15)}$ and the trajectory transfer between sections is optimized. ${ }^{(16)}$ There are two methods of trajectory smoothing: a point cloud smoothing method based on L1 median filtering and a bilateral filtering algorithm. The first method has obvious shortcomings; it will have a large deviation when there are more noise and more outliers. ${ }^{(17)}$ The bilateral filtering algorithm is used for point cloud noise processing because of its good edge retention characteristics, ${ }^{(18,19)}$ but it can only smooth local noise points and takes a long time. In this regard, Xing et al. calculated the Euclidean distance, mean value, and standard deviation between each point in the point cloud and a neighborhood point. ${ }^{(20)}$ Han et al. set the Gaussian distribution threshold to remove outliers and then adopted the guided filtering algorithm to smooth the noise points on the surface of the model. ${ }^{(21)}$ However, this method cannot remove and smooth noise points in the case of sparse model point clouds. Therefore, Liu et al. proposed a Euclidean clustering algorithm under smoothness constraints, which further improved the integrity rate accuracy and detection quality on the basis of Pu et al.'s research. ${ }^{(22,23)}$

The vehicle-mounted point cloud is solved using the position and attitude information collected by the position and orientation system (POS). The quality of the output trajectory 
pose has a direct impact on the accuracy of the vehicle-mounted point cloud. In the past, there were few methods of optimizing a vehicle-mounted mobile measurement system's trajectory data. Generally, a vehicle-mounted mobile measurement system directly calculates the point cloud data from the Kalman filter data jointly calculated by the IMU and GNSS. However, owing to the complex road environment, the vehicle vibration gives the Kalman filtered data local deviations, and it is necessary to further smooth and optimize the obtained trajectory data. To improve the accuracy of the motion trajectory, a vehicle-mounted 3D imaging system generally performs the fast coupling processing of the information obtained by the IMU and GNSS, that is, it uses Kalman filtering to optimize the processing, so that the trajectory is as close as possible to the true motion state. Taking these advances into account, in this paper, we study the movement laws of vehicles from a geometric perspective. Firstly, a model of vehicle motion is constructed according to the time-varying law of the vehicle position and attitude in space. The vehicle trajectory is segmented, and finally, the data is filtered and smoothed by combining SG filtering and B-spline curve filtering. This method can eliminate the sharp transition points caused by the irregular movement of vehicles, which enhances not only the smoothness and continuity of data but also the local characteristics of the motion trajectory.

\section{Error Analysis of Motion of Vehicle Mobile Measurement System}

\subsection{Measurement error analysis}

Many factors affect the accuracy of a point cloud, including placement, positioning, attitude, and coordinate conversion errors. The placement error is a systematic error, which has an impact on the overall point cloud positioning accuracy. In this paper, we do not consider the analysis of this error. The coordinate conversion error has a small effect and can be ignored. We only analyze the impact of the position and attitude information in the trajectory on the point cloud positioning accuracy. According to the positioning principle of a vehicle-mounted 3D imaging system, the position information includes the rectangular spatial coordinates of the origin of the local horizontal coordinate system. The original point cloud data are converted from the laser scanner coordinate system to a geocentric solid coordinate system and then combined with the earth coordinate system. The information is used to determine the conversion matrix of the inertial coordinates to the local horizontal coordinate system. The impact of the error is shown as

$$
\left[\begin{array}{c}
X \\
Y \\
Z
\end{array}\right]_{\text {error }}=\left[\begin{array}{c}
X_{G P S} \\
Y_{G P S} \\
Z_{G P S}
\end{array}\right]+\left[\begin{array}{c}
\Delta X_{G P S} \\
\Delta Y_{G P S} \\
\Delta Z_{G P S}
\end{array}\right]+R_{N}\left[R_{M} \Delta R_{M}\left[R_{L}\left[\begin{array}{c}
x \\
y \\
z
\end{array}\right]+\left[\begin{array}{c}
\Delta x_{L}^{1} \\
\Delta y_{L}^{1} \\
\Delta z_{L}^{1}
\end{array}\right]\right]\right]
$$

In the formula, $\left[\begin{array}{lll}X & Y & Z\end{array}\right]_{\text {error }}$ is the rectangular spatial coordinate with errors, $\left[\Delta X_{G P S} \Delta Y_{G P S}\right.$ $\left.\Delta Z_{G P S}\right]$ is the positioning error, and $\Delta R_{M}$ is the attitude error matrix. The positioning error 
directly affects the position accuracy of the scanning surface and the attitude error indirectly affects the accuracy of the scanning surface. The attitude angle mainly includes the roll, pitch, and heading angles. Taking the $X$-axis of the vehicle movement direction as an example, the roll angle mainly causes changes in $Y Z$ plane coordinates, which have no effect on the $X$-axis of the vehicle movement direction; the pitch angle primarily affects the point coordinates of the $X Z$ plane and does not affect the $Y$ coordinate; the heading angle primarily affects the point coordinates of the $X Y$ plane and does not affect the $Z$ coordinate. The analysis results can be seen in Fig. 1. Figure 1(a) shows the development of the yaw angle error. The $Z$ coordinate of the point cloud does not change and the $X Y$ plane coordinates produce a certain amount of deviation movement. At the same time, the position deviation of the scan line also varies by different values. Figure 1(b) shows the deviation of the point caused by the pitch angle error, which mainly affects the $X Z$ plane coordinates. Figure 1(c) shows the effects of the roll angle error on the accuracy of the point position and the $Y Z$ plane coordinates. The motion estimation error is random, and each trajectory point has a different effect on the pointing accuracy and has a greater impact on the relative accuracy of the vehicle point cloud data. As shown in

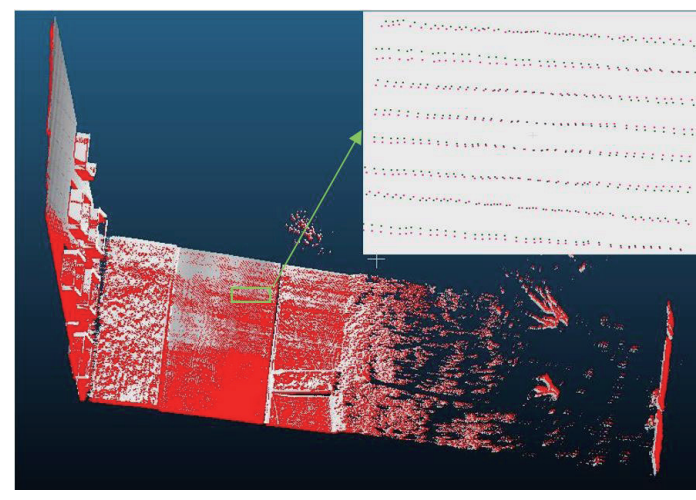

(a)

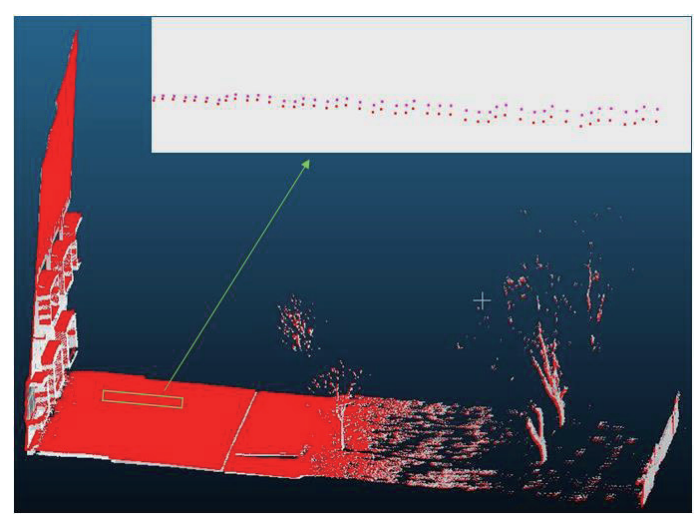

(c)

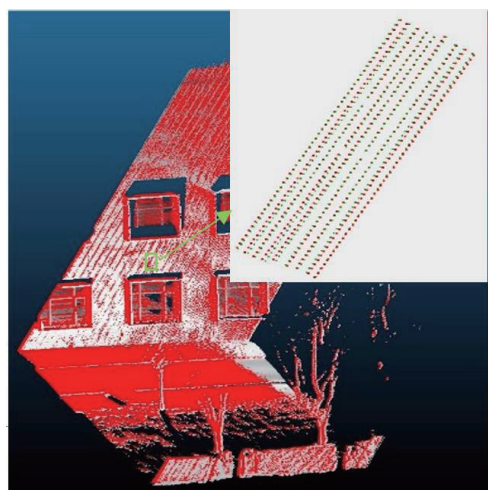

(b)

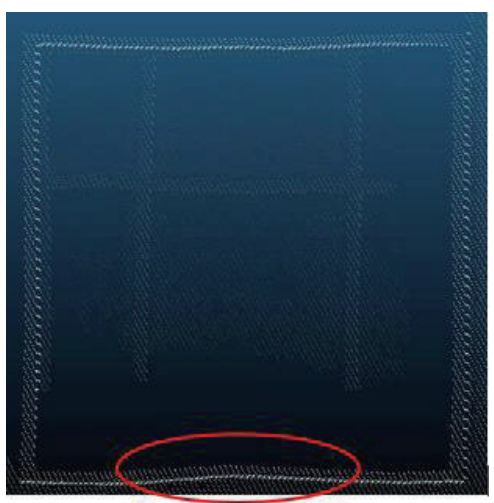

(d)

Fig. 1. (Color) Effect of motion trajectory error on point accuracy. (a) Effect of yaw error on point position; (b) effect of pitch angle error on point position; (c) effect of rolling angle error on point position; (d) effect of motion trajectory error on point accuracy. 
Fig. 1(d), when a vehicle passes through an area such as undulating ground or speed bumps, an instantaneous change will cause the car to vibrate. An inertial navigation element cannot accurately record position and attitude information, resulting in movement trajectory deviation. The recorded track data are not accurate, resulting in the local deformation of the window. Therefore, the motion trajectory should be further optimized before data calculation to eliminate or reduce the effects of position and attitude deviation.

\subsection{Building a motion model}

To analyze the motion state of vehicle motion data, a model of the vehicle motion must be established first. As a multisource sensor fusion system, each sensor of the vehicle-mounted 3D imaging system has its own coordinate system. Therefore, it is necessary to know the coordinate system of each sensor before establishing the same model of earth observation, including the transition coordinate system for coordinate conversion.

\subsubsection{Scanner coordinate system}

The LiDAR scanner coordinate system is divided into a polar coordinate system and a rectangular coordinate system. The polar coordinate system uses the LiDAR emission center as the coordinate origin and is expressed as the distance $\rho$ and rotation angle $\theta$ from the LiDAR emission center to the irradiated object, $(\rho, \theta)$. The rectangular coordinate system is a righthanded coordinate system, which varies with the design of the scanner. The general coordinate origin is the LiDAR emission center, the axis corresponding to the $0^{\circ}$ angle of the laser scanning surface is the $X$-axis, and the axis corresponding to the $90^{\circ}$ angle of the laser scanning surface is the $Y$-axis. The $Z$-axis is perpendicular to the $X O Y$ plane. In this study, a Faro $X-130$ LiDAR scanner is used as the point cloud acquisition device of the vehicle-mounted system, the origin of the coordinates is the laser emission center, the $X$-axis is the initial laser beam emission direction and points to the right side of the vehicle motion direction in the vehiclemounted 3D imaging system, and the $Y$-axis points to the tip of the vehicle in the movement direction, as shown in Fig. 2.

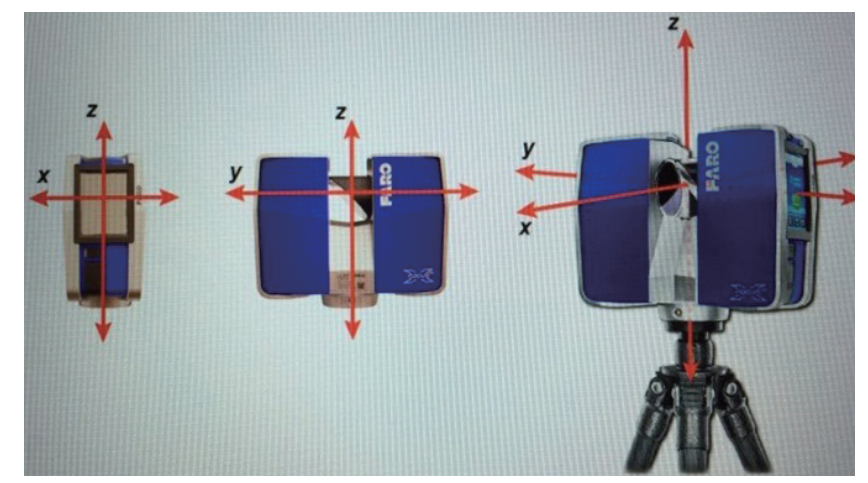

Fig. 2. (Color online) Schematic diagram of laser scanner coordinate system. 


\subsubsection{Inertial navigation coordinate system}

The inertial navigation coordinate system is the coordinate system of the inertial navigation device itself, which is a right-handed rectangular coordinate system. The origin of the coordinate system is located at the center of the inertial navigation sensor, and it is also the intersection point of the three axes of the accelerometer and gyroscope. The axial direction of the coordinate system is consistent with the axial law of the sensor. According to the installation design, the $X$-axis of an inertial navigation system is the same as the moving direction of the vehicle, the $Y$-axis points to the right side of the moving direction of the vehicle, and the $Z$-axis is vertically downward to form a right-handed coordinate system. The attitude observation value is the angle difference between the coordinate system and the local horizontal coordinate system, as shown in Fig. 3.

\subsubsection{Carrier coordinate system}

For the convenience of research and measurement, we set the coordinate system and inertial navigation sensor to the same coordinate system, where the origin is the center of the IMU, the $X$-axis points to the direction of vehicle movement, the $Y$-axis points to the right of the vehicle movement direction, and the $Z$-axis is vertically downward. As a result, coordinate conversion is reduced, unnecessary placement errors are avoided, data processing is simplified, and the work efficiency is improved. The carrier coordinate system is shown in Fig. 4.

\subsubsection{Local horizontal coordinate system}

The local horizontal coordinate system is the reference coordinate system used by the inertial navigation unit to calculate the attitude angle. The attitude angle parameters are determined by

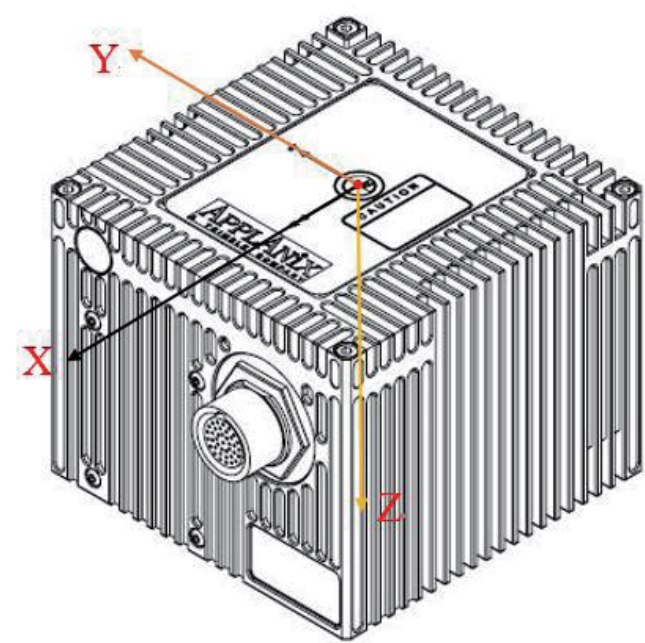

Fig. 3. (Color online) Schematic diagram of laser scanner coordinate system.

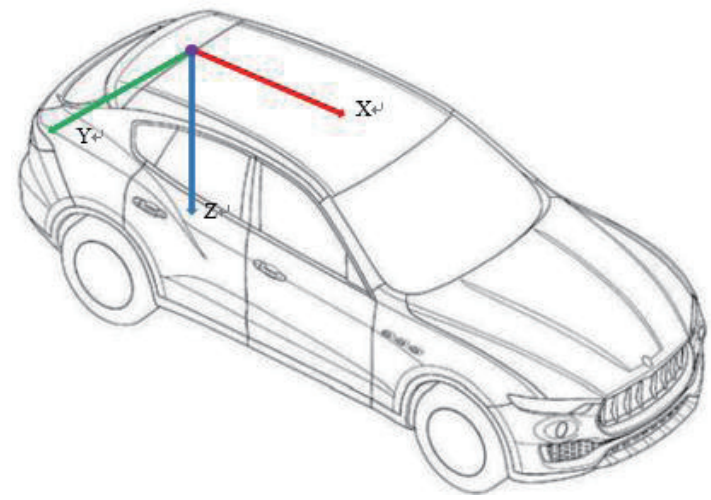

Fig. 4. (Color online) Schematic diagram of carrier coordinate system. 
measuring the angular relationship between the inertial navigation carrier coordinate system and the local horizontal coordinate system. The origin of the coordinates is not fixed and coincides with the center of the sensor, and moves with the movement of the vehicle-mounted platform. The $X$-axis points to the meridian circle direction of the reference ellipsoid, that is, the true north direction, the $Y$-axis points to the direction of the unitary circle, and the $Z$-axis coincides with the normal line. Depending on the rules, the local horizontal coordinate system can be divided into two kinds: northeast sky (ENU) and northeast earth (END). The coordinate system used for attitude calculation in this paper is the ENU coordinate system, as shown in Fig. 5.

\subsubsection{Coordinate transformation mode}

The positioning principle of the vehicle-mounted 3D imaging system is to convert the obtained laser scanning data into the geodetic coordinate system. Its essence is the process of coordinate conversion. The positioning model of the vehicle-mounted 3D imaging system is constructed by combining the laser point coordinates with the acquired position and the attitude information and sensor calibration parameters. Its model is shown as

$$
\left[\begin{array}{c}
X \\
Y \\
Z
\end{array}\right]=\left[\begin{array}{c}
X_{G P S} \\
Y_{G P S} \\
Z_{G P S}
\end{array}\right]+R_{N}\left[R_{M}\left[R_{L}\left[\begin{array}{c}
x \\
y \\
z
\end{array}\right]+\left[\begin{array}{c}
\Delta x_{L}^{1} \\
\Delta y_{L}^{1} \\
\Delta z_{L}^{1}
\end{array}\right]\right]\right] .
$$

Here, $\left[\begin{array}{lll}X & Y & Z\end{array}\right]$ are the laser point cloud coordinates, $R_{L}$ is the rotation matrix from the laser scanner coordinate system to the inertial coordinate system, $\left(\Delta x_{L}^{1}, \Delta y_{L}^{1}, \Delta z_{L}^{1}\right)^{T}$ is the eccentricity from the laser scanner coordinate system to the inertial coordinate system, $R_{M}$ is the conversion matrix from the inertial coordinate system to the local flat coordinate system, $R_{N}$ is the rotation matrix from the local horizontal coordinate system to the Earth-centered Earth-fixed (ECEF) coordinate system, and $\left[X_{G P S} Y_{G P S} Z_{G P S}\right]^{T}$ are the coordinates in the ECEF coordinate system from the origin of the local horizontal coordinate system.

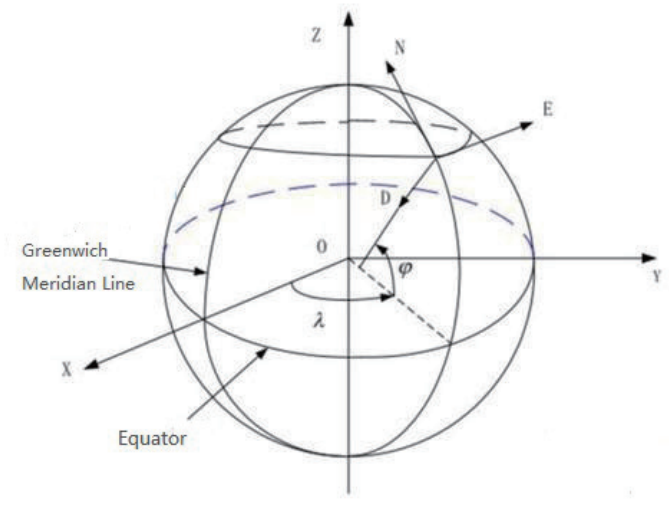

Fig. 5. Schematic diagram of local horizontal coordinate system. 


\section{Processing to Optimize Trajectory}

We propose an optimization method based on previous studies for the vehicle-mounted trajectory data of a mobile LiDAR measurement system. Through the vehicle-mounted mobile measurement system, Kalman filter vehicle-mounted trajectory data based on GNSS and IMU joint solution are obtained. The process is as follows (Fig. 6):

1. Analyze the motion state on the basis of the Kalman filter vehicle trajectory data, establish a vehicle motion model, and determine the motion state and 3D attitude of the vehicle according to the position, speed, motion attitude, and acceleration parameters.

2. Roughly segment the vehicle motion track according to the motion posture, wherein the vehicle turning track is divided into individual parts, and further segment the segments according to the motion acceleration, so that each piece has the same motion direction and the change in motion acceleration is lower than a threshold value.

Using the segmentation results, the intra-segment and inter-segment vehicle-mounted trajectory data are smoothed. Specifically, the intra-segment vehicle-mounted trajectory data are optimized by the polynomial smoothing principle of the least-squares method, and the inter-segment vehicle-mounted trajectory data are optimized by the smoothing code based on B-spline curves to obtain preliminarily optimized vehicle-mounted trajectory data.

\subsection{Self-adaptive threshold segmentation of trajectory}

The vehicle-mounted 3D imaging system uses a vehicle as a carrier to collect point cloud data. The movement speed is high, the data volume is large, and the observation range can reach tens of kilometers. Processing the overall trajectory data requires a large amount of calculation. At the same time, after the vehicle trajectory is filtered, there is a certain deviation in the local area. If all the data are processed, it will increase the amounts of calculation and waste resources. Therefore, before the trajectory is optimized, the rotation is segmented: position and attitude thresholds are determined for each data segment, and the deviated trajectory is searched for and processed to improve the correlation between the data.

Firstly, the motion state is analyzed using the collected vehicle motion data, a model of vehicle motion is established to determine the motion direction of the vehicle according to the

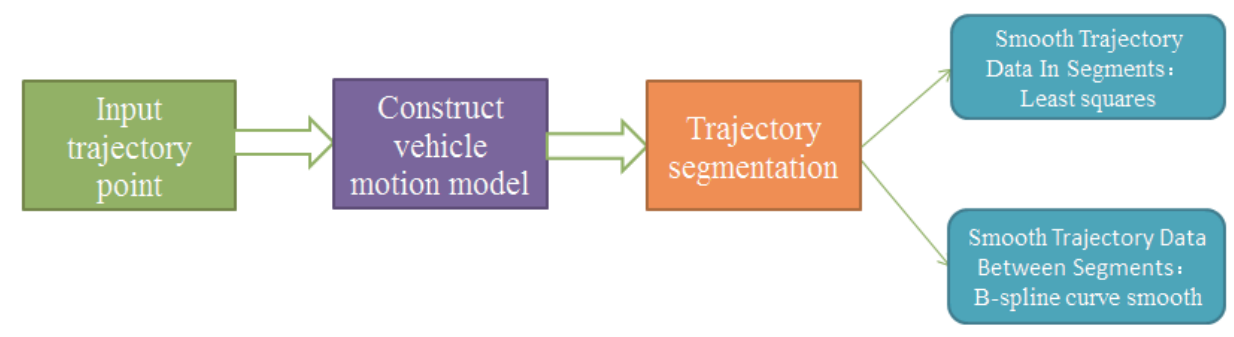

Fig. 6. (Color online) Vehicle trajectory optimization processing flow. 
sign of its speed, the data indicating a small difference change in speed are identified as being from the same region segment according to the acceleration magnitude judgment speed change, and the change in attitude is determined at the same time; the magnitude of attitude and the sign of the speed represent the motion direction of the vehicle and the 3D state of the motion, respectively.

Trajectory segmentation according to the vehicle motion state includes two parts: coarse segmentation and fine segmentation. Coarse segmentation roughly segments the trajectory according to the direction of the motion and the attitude. We divide the same motion directly into the same segments, where the turning area of the vehicle is the same segment alone. The subdivision section is encrypted and divided according to the change in speed, i.e., the acceleration. The specific process is as follows.

\subsubsection{Coarse segmentation}

The status of the vehicle motion is analyzed using the attitude data collected by inertial navigation, and the yaw angle can allow us to intuitively determine the vehicle movement direction. The vehicle movement direction is segmented according to the yaw angle. The same movement direction (the change in the angle does not exceed the threshold) is divided into the same segment, where the vehicle's turning trajectory (the arc continues to change, exceeding the threshold) is divided into separate components. By angle segmentation, the long segment trajectories in the same direction are cut and segmented according to the distance. The segmentation here mainly solves the case where the amount of point cloud data in a certain order is large.

\subsubsection{Subdivision of segments}

When separately segmenting the area where the vehicle turns, i.e., the angle changes continuously, segments will be further segmented according to the acceleration of the motion, i.e., the acceleration value will be icreased. In this way, the motion direction of each component is the same, and the change in the acceleration of the motion is lower than the threshold value. According to the acceleration of the vehicle, the trajectory is finely segmented, as shown in Eq. (3). The speed change threshold is set to $\Delta a$. When the absolute value of the acceleration is less than $\Delta a$, it is considered that the speed of the track point in this area does not change markedly, the motion state is roughly the same, and the trajectory is divided into the same segment with the same speed. When the absolute value is greater than $\Delta a$, it is considered that the change in speed is too large and the segmentation process needs to be performed. In this case, the segmentation point is recorded, and the new acceleration is used as the segmentation point for a new comparison, thereby dividing the trajectory into segments with different speeds. The subdivision section here does not include the area where the vehicle turns, that is, the continuous change in the direction of the vehicle is treated as an independent phenomenon. 


$$
\left\{\begin{array}{lc}
v_{i+1}-v_{i}>\Delta a & \text { Different Segments } \\
v_{i+1}-v_{i} \leq \Delta a & \text { Same Segment }
\end{array}\right.
$$

Here, $v$ is the speed of the vehicle, $i$ represents the time at a certain moment, and $i+1$ is the time at the next moment.

\subsection{Determining fluctuating areas}

The vehicle-mounted 3D imaging system uses the position and attitude information output by the POS system to calculate the point cloud data at a later stage. The quality of this information plays a vital role in the quality of the vehicle-mounted point cloud. To ensure the accuracy of the position and attitude information, it is necessary to optimize the obtained position and attitude information using integrated navigation. The IMU has high precision in the short term and can output very smooth position and attitude information, but there are accumulated errors, so the GNSS is needed to correct these accumulated errors. The obtained GNSS and IMU data are fused by a Kalman filter, and the processed POS information has very good smoothness. According to this characteristic of the POS data, it can be considered that the fluctuation of the position and attitude information acquired in a local range is small, with no change in flat terrain. For contents with large changes, it can be considered that there is local fluctuation noise, which needs secondary optimization.

In order to ensure the correctness of the data, we use discreteness to determine the track information of the local area. The calculated difference between the observed position and attitude data shows that the data in the local area in the ideal state have smaller variations and are more robust. In undulating areas, the data change to a greater extent, with obvious differences, and the data distribution is relatively divergent. There are many measurement indexes for the degree of dispersion. In this paper, the standard deviation is used as the evaluation standard for the degree of dispersion of the motion trajectory, and the dispersion threshold $\sigma_{0}$ is set. The standard deviation of each local segmented data is calculated and compared with the set threshold. Segments with standard deviation exceeding the threshold are determined as areas to be processed, and regions with standard deviation not exceeding the threshold remain unchanged. The calculation is shown as

$$
\left\{\begin{array}{l}
\sigma=\sqrt{\frac{\sum_{i=1}^{N}\left(x_{i}-\bar{x}\right)^{2}}{N} \leq \sigma_{0}} \\
\sigma=\sqrt{\frac{\sum_{i=1}^{N}\left(x_{i}-\bar{x}\right)^{2}}{N}>\sigma_{0}} .
\end{array} .\right.
$$




\subsection{Processing to smooth vehicle trajectory data within and between segments}

\subsubsection{Smoothing of track data in segment}

Using the segmentation results, the vehicle trajectory data within and between segments are smoothed. The on-vehicle trajectory data in the segment are optimized by least-squares polynomial smoothing, and the inter-vehicle trajectory data are optimized by smoothing based on the B-spline curve to obtain preliminary optimized on-vehicle trajectory data. The data in the segment use the least-squares polynomial smoothing. That is, the data in the segment are subjected to a sliding weighted average with a certain window size. The data in the window are the first fitted with a polynomial to determine the polynomial coefficients, which uses the leastsquares method to determine the weight coefficient of each control point. The process is as follows:

- Polynomial fitting

Assuming that the window size is $M=2 N+1$ and each point is $(-n,-n+1, \ldots, 0, \ldots, n-1, n)$, polynomial fitting is performed on the data in the window using Eq. (5) to determine the polynomial coefficients:

$$
y=b_{0}+b_{1} x+b_{2} x^{2}+\cdots+b_{k-1} x^{k-1}
$$

where $N$ is the number of control points, $k-1$ is the degree of the fitted polynomial, $b_{0}-b_{k-1}$ are coefficients, and $x$ is the value of each control point. The $N$ of such an equation forms a $K$-ary linear equation group. The fitting parameters are determined by the least-squares method and expressed in matrix form as

$$
\left(\begin{array}{c}
y_{-n} \\
y_{-n+1} \\
\vdots \\
n
\end{array}\right)=\left(\begin{array}{cccc}
1 & -n & \cdots & (-n)^{k-1} \\
1 & -n+1 & \cdots & (-n+1)^{k-1} \\
\vdots & \vdots & \vdots & \vdots \\
1 & n & \cdots & n^{k-1}
\end{array}\right)\left(\begin{array}{c}
b_{0} \\
b_{1} \\
\vdots \\
b_{k-1}
\end{array}\right)+\left(\begin{array}{c}
e_{-n} \\
e_{-n+1} \\
\vdots \\
e_{n}
\end{array}\right)
$$

- Determination of fitting parameters

The least-squares solution of $\tilde{B}$ is

$$
\tilde{B}=\left(X^{T} \cdot X\right)^{-1} \cdot X^{T} \cdot Y
$$

- Smoothing filter

The filter value of $\tilde{Y}$ is

$$
\tilde{Y}=X \cdot B=X \cdot\left(X^{T} \cdot X\right)^{-1} \cdot X^{T} \cdot Y=P \cdot Y,
$$


where $P=X \cdot\left(X^{T} \cdot X\right)^{-1} \cdot X^{T}, P$ can be understood as the weight coefficient of each control point, $Y$ is the input control point, and the least-squares method is used to determine the weighting coefficient to move. The window-weighted average performs smooth filtering. The data in the segment are filtered by the polynomial fitting method of SG local features, which has good smoothness and enhanced data consistency.

\subsubsection{Smoothing of trajectory data between segments}

In order to ensure the smoothness of data between segments and avoid local jump points, we use a B-spline filter for data filtering and smoothing. Using the mixed-function formula of the B-spline curve and the selected data processing range, recursive processing is performed to determine the B-spline curve basis function, then the position and attitude are smoothed.

- B-spline curve formula

$$
P(u)=\sum_{k=0}^{n} P_{k} B_{k, d}(u) u_{\min } \leq u \leq u_{\max }, 2 \leq d \leq n+1
$$

Here, $P_{k}$ is the input control point. There are $n+1$ numbers. $B_{k, d}(u)$ is the basis function, which represents a polynomial of degree $d-1, d$ is the degree, $u$ is a node, and the local control of the $\mathrm{B}$-spline curve is determined by the basis function defined on the sub-interval in the range of $u$.

- Recursion of basis functions

The basis function of the B-spline curve is defined by the Cox-de Boor recursive formula. Its formula is as follows:

$$
\begin{gathered}
B_{k, 1}(u)= \begin{cases}1 & u_{k} \leq u \leq u_{k+1} \\
0 & \text { theother }\end{cases} \\
B_{k, d}(u)=\frac{u-u_{k}}{u_{k+d-1}-u_{k}} B_{k, d-1}(u)+\frac{u_{k+d}-u}{u_{k+d}-u_{k+1}} B_{k+1, d-1}(u) .
\end{gathered}
$$

Each basis function is defined on the $d$ subintervals of the value range of $u$, the selected group of subinterval endpoints $u_{j}$ is a node, and the selected group of subinterval endpoints becomes the node vector as a whole, which can be chosen to satisfy $u_{j} \leq u_{j+1}$. Any value of $(j+1)$ is taken as the endpoint of the subinterval. The maximum and minimum values of $u$ depend on the number of selected control points and $d$.

- Determination of node vector

Assuming that there are $n+1$ control points and $m+1$ node vectors, the degree is $p . n, m$, and $p$ must satisfy $m=n+p+1$. To define a $p$-degree $\mathrm{B}$-spline curve with $n+1$ control points, it is necessary to provide $n+p+2$ nodes $u_{0}, u_{1}, \ldots, u_{n+p+1}$. On the other hand, if a node vector of $m+1$ nodes and $n+1$ control points is given, the number of B-spline curves is $p=m-n-1$, and the nodes divide the B-spline curves into curve segments. 
Finally, the data are subjected to multiple iterative smoothing processes, the processed results are sequentially assigned to corresponding positions, and the obtained new data are taken as input values to be subjected to the required number of multiple iterations according to step 1. As a result, the consistency of the data is enhanced, the local fluctuation of the slope is eliminated, and thus the precision of the data is improved.

\section{Experiment and Analysis}

\subsection{Smooth processing of vehicle trajectory data within and between segments}

Our experiment uses an independent integrated vehicle-mounted 3D imaging system, as shown in Fig. 7. The experimental site is at Beijing University of Civil Engineering and Architecture. First, the onboard scanning route is formulated according to the road to be scanned. The on-board scanning route requires that the vehicle travels as straight as possible. In order to reduce the occlusion of road point cloud data, the point cloud data must be collected when the road is open and there are few vehicles and pedestrians. Before the experiment starts, we need to found an open area around the collection section for static POS scanning. For vehicle-mounted mobile 3D laser scanning, a base station must be established on the control point synchronously. After the data collection is completed, the track is processed in sections.

\subsection{Smoothing of trace}

The original vehicular point cloud converts 2D point coordinates to geodetic coordinates according to the motion trajectory obtained by inertial navigation. The motion trajectory error has an intuitive effect on the accuracy of the point cloud. In order to ensure the accuracy of the data and improve the accuracy of the on-board point cloud, the trajectory must be optimized

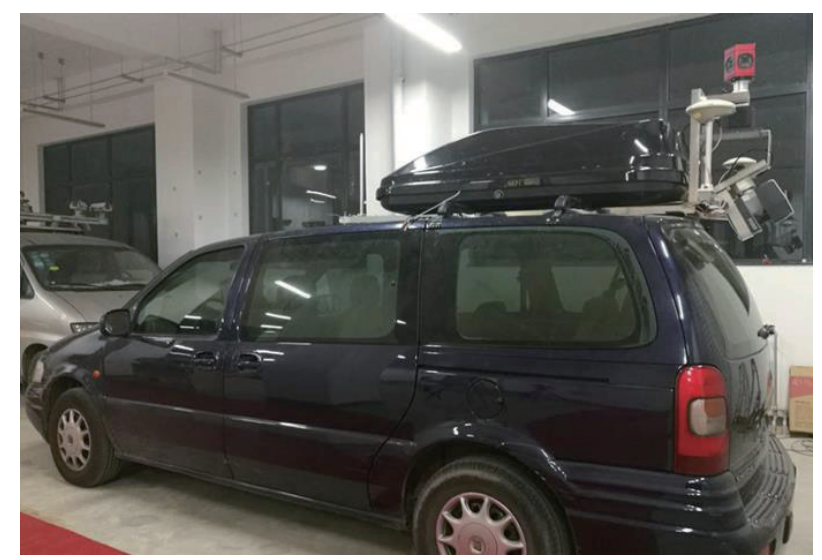

Fig. 7. (Color online) Newly developed vehicle and mobile measurement system. The mobile measurement system is composed of laser scanners, the GNSS global positioning system, the IMU inertial navigation sensor, an odometer, a panoramic camera, and other sensor elements. 
by the above methods. First, we need to segment the trajectory. The attitude and velocity information contained in the trajectory data is considered during segmentation, and then segmentation is carried out according to changes in angle and velocity. Some segmentation results are shown in Fig. 8.

After the trajectory is segmented, the position and attitude information of each segment are analyzed, the dispersion of each data segment is calculated, the calculated standard deviation is compared with a set threshold value, and segments exceeding the threshold value are considered as areas with large fluctuations. The threshold value is set at 0.04 , and an area with a large fluctuation is shown in Fig. 9. Figure 9(a) shows the trajectory data acquired by the vehiclemounted 3D imaging system, Fig. 9(b) shows the result of screening fluctuating regions based on the calculated point cloud data, and Fig. 10 shows the result of screening the trajectory using the threshold setting method. It can be seen from the figure that the segmented points in the problematic regions have been screened out with high accuracy.

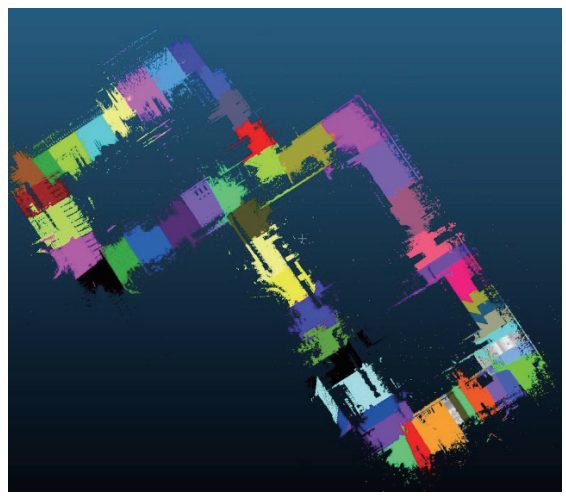

(a)

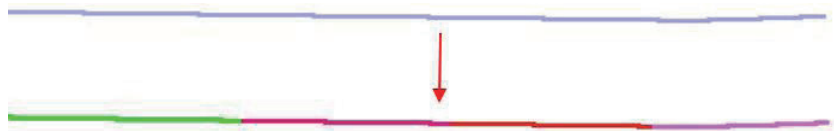

(b)

Fig. 8. (Color) Schematic diagram of trajectory segmentation.

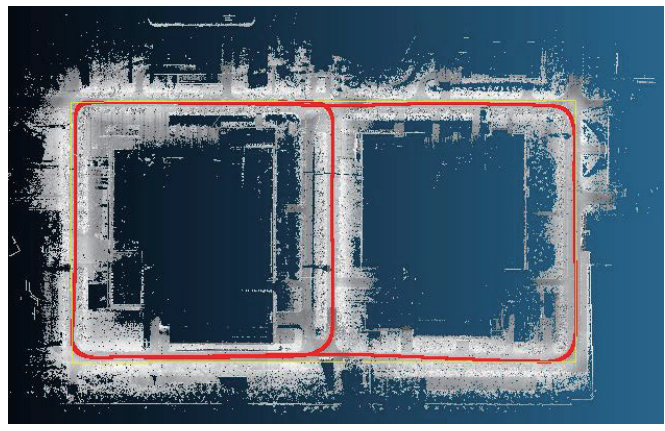

(a)

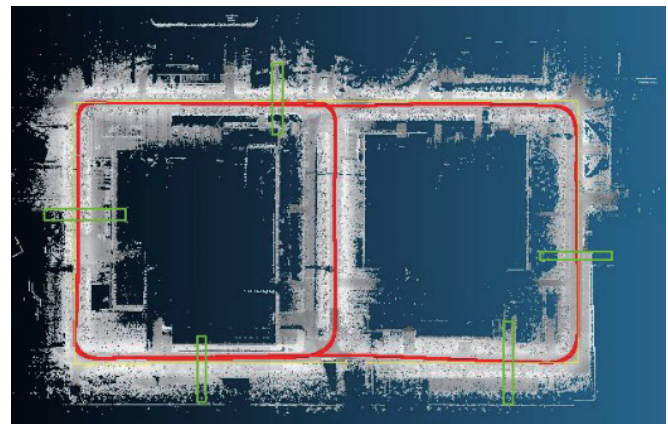

(b)

Fig. 9. (Color) Screening of local undulating area based on point cloud data. (a) Raw trajectory and point cloud data. (b) Results of manually filtering local undulations. 


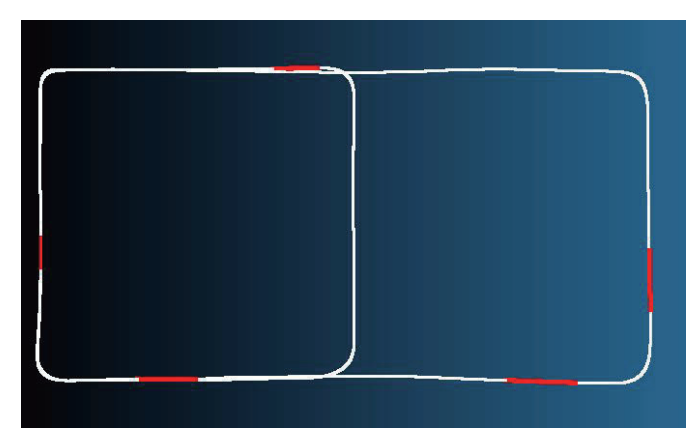

Fig. 10. (Color) Results of automatic screening of undulating area based on trajectory.

\subsection{Comparative analysis of results}

The vehicle motion trajectory is optimized using the segmentation results. To optimize the motion trajectory including the position and attitude, SG filtering and B-spline smoothing are used to process the trajectory jointly. As trajectory optimization improves the position accuracy of the point cloud, the effect of optimization is directly compared and analyzed in terms of the point cloud. Figure 11 shows a comparison of the local positions obtained before and after optimization. It can be seen that there is a clear difference before and after optimization. After optimization, the local undulation in the middle of the window is eliminated and the edge of the window also exhibits a regional horizontal effect, similar to the actual situation. By comparing the precision of the optimized vehicle-mounted point cloud data and the ground point cloud data, we demonstrate the effectiveness of the optimization.

The accuracy of the local deviation of the vehicle-mounted point cloud caused by trajectory noise is analyzed to verify the correctness of the relationship within the point cloud data (Fig. 12). We use indicators such as street lamp spacing, window length, and sign height. As the standard for measuring the accuracy, the true values of these features are obtained using a ground laser scanner with an accuracy of $2 \mathrm{~mm}$. The accuracy for each indicator is shown in Table 1. By selecting multiple spacings for comparison, the accuracy is calculated to be $0.013 \mathrm{~m}$.
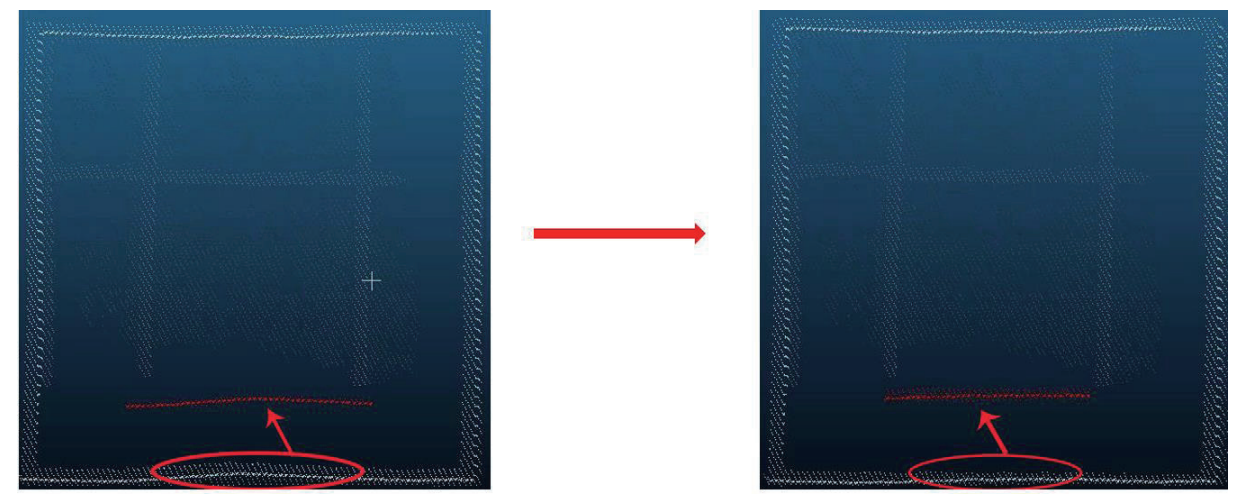

Fig. 11. (Color) Comparison of point cloud results obtained before and after optimization. After optimization, the local undulation in the middle of the window is eliminated and the window edge also exhibits a regional horizontal effect. 


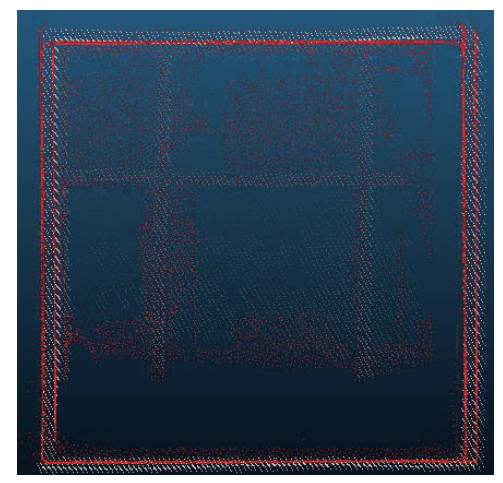

(a)

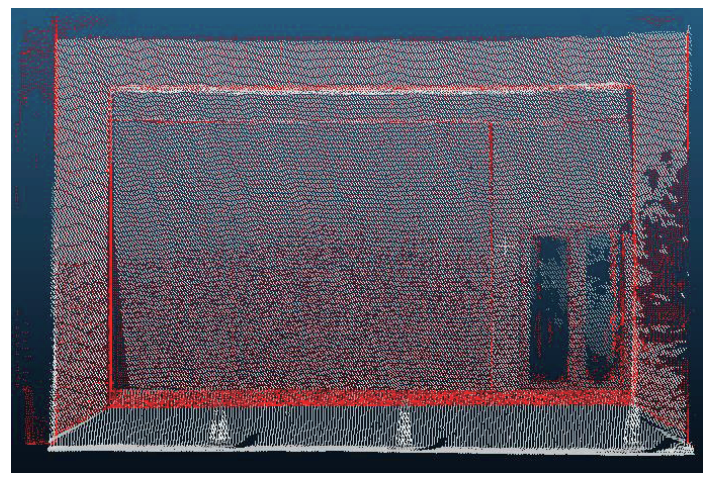

(b)

Fig. 12. (Color) Comparison of point cloud between vehicle LiDAR and ground LiDAR. The vehicle-mounted and ground point clouds similarly describe the ground objects, the geometric shape of the ground objects is unchanged, and the accuracy is high.

Table 1

Comparison of accuracy results.

\begin{tabular}{lccc}
\hline Indicator & $\begin{array}{c}\text { Car point cloud data } \\
(\mathrm{m})\end{array}$ & $\begin{array}{c}\text { Station-borne point cloud data } \\
(\mathrm{m})\end{array}$ & $\begin{array}{c}\text { Error } \\
(\mathrm{m})\end{array}$ \\
\hline Window width & 2.540 & 2.533 & 0.007 \\
Street lamp pitch & 32.342 & 32.357 & -0.015 \\
Building director & 74.773 & 74.785 & -0.012 \\
Building width & 19.393 & 19.404 & -0.011 \\
Road signs & 1.134 & 1.123 & 0.011 \\
\hline
\end{tabular}

\section{Discussion}

Vehicle-mounted 3D imaging systems play an important role in urban data updating, the development of smart construction, and 3D topographic map drawing through the acquisition of 3D ground space information. The systems are composed of many sensors, and the data obtained by individual sensors are fused using time and space registration methods. The accuracy of the point cloud is affected by many factors, such as the sensors themselves and the setting error. Among these factors, the placement and positioning errors have a particularly strong effect. To eliminate the placement error, accurate sensor parameters are obtained by high-precision calibration methods. However, the inevitable positioning and attitude determination errors affect the trajectory accuracy of a vehicle-mounted 3D imaging system and cause the positional deviation of the point cloud. Therefore, to expand the range of applications of our vehicle-mounted 3D imaging system, the accuracy of the point cloud needs to be improved.

The research content of this paper is supported by our developed integrated vehicle 3D imaging system. By analyzing the principles and sources of system errors, an optimization method for smoothing the vehicle trajectory is proposed. In this paper, a model of vehicle motion is proposed, and then the changing trend of the vehicle trajectory with time is analyzed. 
Finally, the vehicle trajectory is segmented using speed and attitude information. In this way, the motion state of the vehicle can be intuitively understood. SG filtering and B-spline curve filtering are combined to filter and smooth the data. This can enhance the smoothness of the data, eliminate the existing local errors, and improve the accuracy of the trajectory data.

Note that after the rough segmentation of the motion trajectory, the long segment trajectory needs to be cut and segmented according to the distance moved so that the value of each segment of the distance is lower than the threshold, and then the angle segmentation is performed. As a result of the angle segmentation, the long segment trajectories in the same direction are cut and segmented according to the distance. The segmentation here mainly solves the case of a large amount of data obtained by driving in a certain direction. In the coarse section, a continuous change in the yaw angle of the vehicle movement posture exceeding $20^{\circ}$ is considered as the vehicle turning and is divided into separate sections.

The segmentation threshold determines the granularity of the trajectory segmentation. Many studies on trajectory segmentation tend to be user-defined in the process of threshold setting, and its defects are mainly reflected in two aspects. First, it increases the level of difficulty for users to determine the optimal segmentation threshold. Second, the same segmentation threshold is used for trajectory data with a different point cloud density, making the segmentation result unsatisfactory. In this experiment, the threshold is set to 0.04 , and a segment with fluctuations that exceed the threshold is considered as a region with a large fluctuation. A method of determining the point of trajectory segmentation that considers the segmentation needs of users and the complexity of the trajectory requires further research.

\section{Conclusions}

In order to optimize the trajectory of a vehicle-mounted system and improve the accuracy of the point cloud, we propose a method of improving the accuracy of the point cloud used for the trajectory optimization of a vehicle-mounted 3D imaging system and the correction of the consistency of the round-trip deviation. The vehicle trajectory data based on the joint solution of the GNSS and IMU is obtained through the vehicle mobile measurement system, and the motion state is analyzed using the Kalman filter vehicle trajectory data to establish a model of the vehicle motion. The vehicle motion trajectory is roughly segmented according to the motion posture, where the vehicle turning trajectory is divided into individual segments. On the basis of the acceleration of the motion, the segments are further subdivided so that the motion direction of each segment is the same and the change in the acceleration of the motion is lower than a threshold. Using the segmentation results, the vehicle trajectory data within and between segments are smoothed to obtain the optimized vehicle trajectory data. Experiments show that this method can improve the smoothness of the data, eliminate existing local errors, and improve the accuracy of the trajectory data. It provides a reference method of improving the accuracy of point clouds and improves the automation process and intelligence of vehicle point cloud processing.

The paper makes important contributions to the improvement of the vehicular point cloud trajectory accuracy. These methods have achieved good results in improving the accuracy of 
vehicle-mounted point clouds, but certain problems need to be further addressed. For example, we need to analyze the effect of the posture error on the accuracy of the point cloud data under different road conditions and further optimize the motion trajectory.

We are currently carrying out further research on a method of correcting the position deviation of the overlapping area of the vehicle-mounted point cloud, and a point correction method that is superior to multiconstraint joint registration is being explored, which will improve the accuracy of point correction and save time. For example, the ICP algorithm can be used for further optimization and to improve the registration results, to obtain more accurate rigid transformation parameters, and further improve the repeatability of the vehicle-mounted point cloud.

\section{Acknowledgments}

This work was supported by the National Natural Science Foundation of China (41971350), the Beijing Advanced Innovation Center for Future Urban Design Project (UDC2019031724), the Development Project of Beijing Municipal Science and Technology (KM201810016013), Fundamental Research Funds for Beijing Universities (X18050, X18247), the Special Entrustment Project of Scientific Research Fund of Beijing University of Civil Engineering and Architecture (KYJJ2017024), Beijing Institutions of Higher Learning Innovation Ability Enhancement (TJSH20161001602), and the Pyramid Talent Training Project Lead Teacher Support Plan (JDJQ20200307). This research was funded by the Fundamental Research Funds for Beijing University of Civil Engineering and Architecture (grant no. X20088), the Postgraduate Innovation Project (PG2020082), (PG2020075).

\section{References}

1 W. Li, M. Burrow, and N. Metje: IEEE Access 8 (2020) 94643. https://doi.org/10.1109/ACCESS.2020.2994470

2 V. Ilci and C. Toth: Sensors 20 (2020) 899. https://doi.org/10.3390/s20030899

3 S. Wei and L. Wang: Spatial Sci. 5 (2020) 1. https://doi.org/10.1080/14498596.2020.1791271

4 X. Xu, L. Zuo, X. Li, L. L. Qian, J. K. Ren, and Z. P. Sun: IEEE Trans. Syst. Man Cybern. Syst. 50 (2018) 3884. https://doi.org/10.1109/TSMC.2018.2870983

5 A. M. Samad: Control \& System Graduate Research Colloquium (IEEE, 2016). https://doi.org/2016.10.1109/ ICSGRC.2016.7813329

6 Z. Yiming, L. Yanhua, and S. Yanan: J. Teach. Res. Chem. 35 (2014) 4. https://doi.org/10.13435/j.cnki. ttc. 002649

7 J. Jin: Remote Sens. 12 (2020) 209. https://doi.org/10.3390/rs12020209

8 W. Yulin: J. Geomatics 41 (2016) 86. https://doi.org/ 10.14188/j.2095-6045.2016.03.021

9 R. D. Williams: Earth Surf. Processes Landforms 7 (2020) 251. https://doi.org/10.1002/esp.4747

10 E. Che, M. J. Olsen, C. E. Parrish, and J. Jung: Photogramm. Eng. Remote Sens. 85 (2019) 573. https://doi. org/10.14358/PERS.85.8.573

11 W. Li, M. Burrow, and N. Metje: IEEE Access 99 (2020) 1. https://doi.org/10.1109/ACCESS.2020.2994470

12 L. Yun: Geospatial Inf. 4 (2016) 24. https://doi.org/10.3969/j.issn.1672-4623.2016.04.008

13 C. Liang: Opt. Lasers Eng. 51 (2013) 493. https://doi.org/10.1016/j.optlaseng.2012.10.010

14 X. D. Chen: Opto-Electron. Rev. 7 (2019) 28. https://doi.org/10.12086/oee.2019.190182

15 W. Fan, C. H. Lee, and J. H. Chen: Int. J. Mach. Tools Manuf. 96 (2015) 27. https://doi.org/10.1016/ j.ijmachtools.2015.04.009

16 Y. Liu, K. Xiong, Q. Ni, P. Y. Fan, and L. K. Ben: IEEE Internet Things J. 7 (2019) 2777. https://doi. org/10.1109/JIOT.2019.2958975 
17 C. Hu, Z. Pan, and P. Li: Remote Sens. 11 (2019) 2. https://doi.org/10.3390/rs11020198

18 X. Shi, T. Liu, and X. Han: Int. J. Remote Sens. 41 (2020) 8. https://doi.org/10.1080/01431161.2019.1701211

19 S. Jia, G. Zhang, and X. Zhang: 9th Int. Conf. Graphic Image Process. (ICGIP, 2018). https://doi. org/10.1117/12.2303607

20 C. Xing, Z. Wang, and F. Meng: IET Comput. Vision. 13 (2019) 44. https://doi.org/10.1049/iet-cvi.2018.5027

21 X. F. Han, J. S. Jin, and M. J. Wang: Signal Process.: Image Commun. 57 (2017) 10. https://doi.org/10.1016/ j.image.2017.05.009

22 R. F. Liu, X. S. Liu, and G. W. Yue: Geomatics Inf. Sci. Wuhan University 42 (2017) 250. https://doi. org/10.13203/j.whugis20140959

23 D. Pu, M. Rutzinger, and Vosselman: Isprs J. Photogramm. Remote Sens. 66 (2011) S28. https://doi. org/10.1016/j.isprsjprs.2011.08.006 